\title{
Mycobacterium malmoense infections in Scotland: an increasing problem
}

\author{
A J FRANCE, D T McLEOD, M A CALDER, ANTHONYSEATON \\ From the Chest Unit and Department of Microbiology, City Hospital, Edinburgh
}

ABSTRACT During 1982-4 20 cases of Mycobacterium malmoense infection were identified in Scotland (13 male, seven female; age 34-82, median 62 years). Features of the disease were obtained from case notes and radiographs of 19 patients and were found to be indistinguishable from those of patients with pulmonary tuberculosis. Chronic chest disease, predominantly chronic airflow obstruction, was the most frequent associated disease. The organisms showed in vitro resistance in eight patients to rifampicin, in 19 patients to isoniazid, and in all patients to pyrazinamide and $p$-aminosalicylic acid. Nevertheless, all patients showed an early response to standard combination chemotherapy with rifampicin, isoniazid, and ethambutol, with or without pyrazinamide. Five have been cured and none has died of the infection, although four died of unrelated disease. Of nine patients still having treatment, five had relapsed after completing a course of antituberculosis drugs. All had received ethambutol for less than five months. The response to standard drugs was more satisfactory when the course included administration of ethambutol for at least nine months. Currently one new infection with $M$ malmoense occurs in Scotland for every 40 with tuberculosis, and the incidence appears to be rising. In view of this, it is suggested that when tuberculosis is suspected the chemotherapeutic regimen should include ethambutol until the culture results are reported. If these then show $M$ malmoense, ethambutol should be continued in the combination for at least nine months.

\section{Introduction}

Myobacterium malmoense was first described as a respiratory pathogen in $1977 .^{1}$ Since then there have been reports from the United Kingdom ${ }^{2-4}$ and from North America. ${ }^{5}$ The original report from Sweden described the in vitro sensitivities of the organism: it was sensitive to ethambutol, cycloserine, ethionamide, and kanamycin but resistant to isoniazid, p-aminosalicylic acid, rifampicin, and streptomycin. The authors commented on the particularly slow growth of the organism, which formed tiny colonies on standard mycobacterial culture media. Biochemical features and the unique lipid pattern revealed by thin layer chromatography distinguish the organism from other species of mycobacteria. An early report from Cardiff noted a high prevalence of pneumoconiosis in the 11 patients described. ${ }^{2}$

We describe our experience of this organism in

Address for correspondence: Dr AJ France, Infectious Diseases Unit, City Hospital, Edinburgh EH10 5SB. (Reprints will not be available.)
Scotland and discuss the problems encountered with chemotherapy.

\section{Methods}

Cultures from all over Scotland are sent to the mycobacterium reference laboratory at the City Hospital for identification. Our identification criteria for $M$ malmoense are: (1) microaerophilia; (2) growth at $25^{\circ}$ and $37^{\circ}$ but not at $45^{\circ} \mathrm{C}$; (3) ability to hydrolyse polyoxyethylene sorbitan esters of fatty acids (Tween); (4) a characteristic lipid pattern on thin layer chromatography.

In those cases where $M$ malmoense was isolated we requested the case notes and radiographs from the hospital concerned. Summaries of the history and examination findings were prepared by two of the authors. Another author reported the chest radiographic appearances without prior knowledge of the case histories or clinical progress of the patients.

\section{Results}

During the three years 1982-4 inclusive cultures of 
samples from 20 patients with chest disease were identified as $M$ malmoense. Case notes and radiographs were obtained from 19.

Eighteen presented with cavitating pulmonary infection and one with a pleural effusion. There was a wide age range, 34-82 years, but most patients were over 60 (median 62). The disease was twice as common in men (13 patients) as in women (seven patients). Symptoms of cough and weight loss were present in three quarters of the patients. The clinical and radiological features were indistinguishable from those of pulmonary tuberculosis.

Other, possibly predisposing, diseases were identified in most patients. Seventeen were cigarette smokers. Fifteen had a past history of chronic respiratory disease, predominantly chronic airflow obstruction (nine patients). Other respiratory disease included two cases of recurrent pneumothoraces and one each of pulmonary fibrosis, thoracoplasty for tuberculosis, invasive aspergillosis, and pneumoconiosis. Two patients were having long term corticosteroid treatment and four had coexisting malignant disease (breast, prostate, Waldenström's macroglobulinaemia, and carcinomatosis). Eight patients had a history of peptic ulceration, three having been treated by surgery.

All isolates were tested in vitro with a wide variety of antimycobacterial antibiotics. A summary of the results is shown in the figure. All strains were resistant to pyrazinamide and $p$-aminosalicyclic acid. Only one was sensitive to isoniazid and 12 were sensitive to rifampicin (figure).

The drug regimen in all patients was a standard combination of first line drugs (rifampicin, isoniazid, and ethambutol with or without pyrazinamide), decisions on treatment having been made by individual physicians looking after the patients. The outcomes may be divided into four groups as follows:

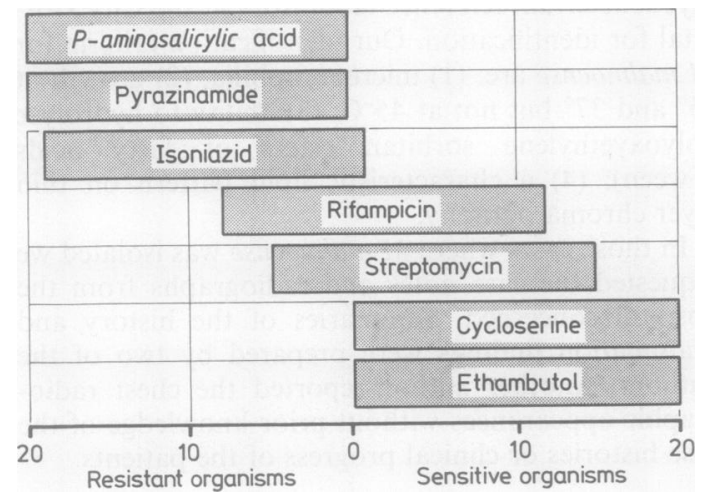

Spectrum of drug resistance: in vitro sensitivities in 20 cases of Mycobacterium malmoense infection.
1 Cure Five patients have been bacteriologically $\overrightarrow{\vec{F}}$ cured. Four patients received first line drugs alone $;$ another patient had second line drugs (streptomycin $\frac{\mathrm{C}}{0}$ prothionamide, and clofazimine) added when the in $\overline{\bar{n}}$.

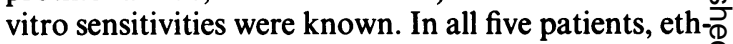
ambutol was used throughout the period of treatment (mean 15, range 9-21 months).

2 Relapse Five patients relapsed after completing $\overrightarrow{0}$ a standard course of combination treatment. Eth- $=$

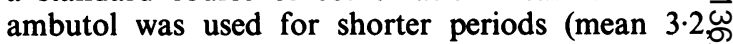
range 2-4 months). Chemotherapy was restarted between two months and two years of completing the initial course of treatment, the patients receiving respectively (a) rifampicin, isoniazid, cycloserine ${ }_{i}^{\infty}$ clofazimine, and prothionamide; (b) rifampicin ethambutol, isoniazid, and pyrazinamide; $(c)$ rifamo picin, ethambutol, isoniazid, and cycloserine; $(d)_{-}^{2}$ rifampicin, ethambutol, isoniazid, and streptomycin (e) rifampicin, ethambutol, isoniazid, streptomycin and thiacetazone. All five patients improved on restarting chemotherapy and their condition remains. stable.

3 Death during treatment Four patients, mean age 71 years, died of unrelated causes (myocardiap infarction in two cases, bronchopneumonia, and cars cinomatosis) before completing their course of treat ment.

4 Still having treatment Four patients had yet tळ finish their course of treatment at the time of this study but their condition was stable.

One further patient was lost to follow up.

\section{Discussion}

It is not surprising that these patients were treated as having tuberculosis for the first two months. The clini ical presentation gives no clue to the nature of the mycobacterium responsible for the disease. The slow growth of the organism in culture is the first pointer in favour of an atypical mycobacterium but this may not be apparent for at least eight weeks. In laboratories. where "negative" cultures are discarded after si weeks of incubation the diagnosis of $M$ malmoens may be missed and the patient erroneously labelled as having "culture negative tuberculosis." Our practice is to keep "negative" cultures for eight weeks before discarding them. Smear positive but culture negative samples are kept for a further four weeks.

Current short course chemotherapy relies on treatment by administration of three or four drugs to which the mycobacteria are sensitive. The drug regit mens made use of rifampicin, isoniazid, ethambutof and, in six month regimens, pyrazinamide. Of these drugs, only ethambutol is bacteriostatic in vitro for a strains of $M$ malmoense. Rifampicin resistance was 
present in $40 \%$ and isoniazid resistance in $95 \%$ of our 20 patients. Despite this resistance pattern, all patients made a good initial response to standard antituberculosis drug regimens. The in vitro resistance patterns of $M$ malmoense to individual first line antituberculosis drugs are different from the patterns observed when the drugs are tested in combination. ${ }^{6}$ In particular, the combination of rifampicin and ethambutol reduces the minimum inhibitory concentration of each drug to the lowest value normally used in sensitivity testing. In a review of drug treatment of $M$ malmoense infections in England and Wales, the most favourable responses were noted in those patients who received prolonged courses of ethambutol combined with other first line drugs. ${ }^{7}$ Our observations support this view. The mean duration of ethambutol treatment was 15 months in the patients who were cured compared with only 3.2 months in those who relapsed.

Our experience has not shown any association with pneumoconiosis, which contrasts with an earlier report from Cardiff. ${ }^{2}$ Many coalminers with pneumoconiosis have chronic airflow obstruction as well, and as many of our patients had this condition possibly the association is with this rather than with pneumoconiosis. Pneumoconiosis is relatively uncommon in Scotland and the likelihood of a chance association is correspondingly low.

With the decline of tuberculosis in Scotland from 1303 notifications in 1978 to 726 in 1985, $\mathrm{M}$ malmoense and other non-tuberculous mycobacteria are responsible for an increasing proportion of mycobacterial infection. It is noteworthy that our laboratory has identified 18 new cultures of $M$ malmoense infection from Scotland in 1985, when there were 726 notifications of tuberculosis, a ratio of 1:40. The annual incidence of $M$ malmoense in the UK has been rising since the beginning of this decade, böth in absolute terms and in relation to tuberculosis. ${ }^{8}$ There is no obvious explanation for this rise. ${ }^{9}$ The population of Scotland is 5.1 million, about one tenth of the population of England and Wales. If the incidence of $M$ malmoense infection were the same in England and Wales as in Scotland, we would expect about 180 patients to have been reported in 1985. This has not occurred, so we must assume either that the disease is underdiagnosed south of the border or that there is some epidemiological factor that makes it more common in Scotland.

To maximise the possibility of cure, the wise policy is to continue a triple drug regimen that includes ethambutol in all patients suspected of tuberculosis until the drug sensitivities are reported. If $M$ malmoense is reported, our practice would be to treat the patient for at least a year with the three drugs rifampicin, isoniazid, and ethambutol. Bacteriological follow up, at least until more information is available on the long term results, should be continued indefinitely. At present it is not possible to give firm guidance on the management of relapse, though results in Scotland so far indicate that the disease is still responsive to first line drugs provided that these include ethambutol.

We are most grateful to Mr Alan Rayner for performing the thin layer chromatography. Data on numbers of tuberculosis notifications were provided by the Communicable Diseases Unit, Ruchill Hospital, Glasgow.

\section{References}

1 Schroder KH, Juhlin I. Mycobacterium malmoense sp nov. Int J Systematic Bacteriol 1977;27:241-6.

2 Jenkins PA, Tsukamura M. Infections with Mycobacterium malmoense in England and Wales. Tubercle 1979;60:71-6.

3 Barclay J, Stanbridge TN, Doyle L. Pneumonectomy for drug resistant Mycobacterium malmoense. Thorax 1983;38:796-7.

4 Roberts C, Clague H, Jenkins PA. Pulmonary infection with Mycobacterium malmoense: a report of 4 cases. Tubercle 1985;66:205-9:

5 Warren NG, Body BA, Silcox VA, Matthews JH. Pulmonary disease due to Mycobacterium malmoense. $J$ Clin Microbiol 1984;20:245-7.

6 Banks J, Jenkins PA. The effect of combined versus single anti-tuberculosis drugs on in vitro sensitivity patterns of $M$ malmoense and $M$ xenopi. Thorax 1985;40:697-8.

7 Banks J, Jenkins PA, Smith AP. Pulmonary infection with Mycobacterium malmoense-a review of treatment and response. Tubercle 1985;66:197-203.

8 Grange JM, Yates MD. Infections caused by opportunist mycobacteria: a review. $J R$ Soc Med 1986;79:226-9.

9 Jenkins PA. Mycobacterium malmoense. Tubercle 1985;66:193-5. 